\title{
La libération, un outil puissant pour optimiser le volume des déchets radioactifs
}

\author{
W. BLOMMAERT*
}

(Manuscrit reçu le 6 avril 1998, révisé le 7 juillet 1998, accepté le 4 novembre 1998)

RÉSUME Dans le cadre de ses activités de déclassement d'installations nucléaires, Belgoprocess met en ceuvre la libération pour optimiser les volumes des déchets radioactifs. La méthodologie de libération retenue est discutée ici; on porte une attention spéciale aux différents types de matériaux et aux mesures associées. Trois exemples concrets sont abordés : ils traitent le déclassement et la décontamination de deux petits bâtiments, et ce jusqu'au stade du « green field »; la fusion et la libération d'acier inoxydable constituant des dispositifs de stockage de combustible ( rack $\leadsto)$; l'aluminium issu d'échangeurs de chaleur. Une attention particulière est portée aux problèmes pratiques associés, aux stratégies d'échantillonnages et de mesures, ce en relation avec les limites basses proposées pour la libération inconditionnelle.

ABSTRACT Clearance, a powerful tool in optimizing the volume of radioactive wastes

In the framework of its activities in decommissioning nuclear installations, Belgoprocess applies the clearance practice in optimizing the volume of radioactive wastes. The applied clearance methodology is discussed with special emphasis to different types of materials and to the associated measurements. Three practical cases are elaborated, dealing with the decommissioning and decontamination of two small building up to green field conditions, the melting and clearance of stainless steel fuel racks, and of aluminium heat exchangers. The attention is drawn on pratical problems associated with sampling and measurement strategies, in relation to the proposed low limits for unconditional release.

\section{Introduction}

En Belgique, Belgoprocess peut être considéré comme un pionnier dans le démantèlement et l'application du principe de libération à l'échelle industrielle. Un projet pilote de déclassement a été réalisé de 1988 à 1991; il inclut le démantèlement et le retrait de toute zone contrôlée de deux bâtiments de l'entreprise de retraitement

* Belgoprocess NV, Gravenstraat 73, 2480 Dessel, Belgium. 
appelée alors Eurochemic. Le projet a démontré, notamment, la faisabilité de la décontamination de composants et de structures en béton jusqu'à des niveaux permettant une libération inconditionnelle.

Les premières étapes vers la mise en pratique effective de la libération inconditionnelle furent toutefois difficiles car la nécessité de convaincre les autorités de l'application d'une libération inconditionnelle était réelle. L'absence de toute législation belge (nucléaire) pour le réemploi sans restriction et le rejet incontrôlé, de matières suspectes et/ou décontaminées, combiné à l'absence de limites de rejet inconditionnel au niveau international à cette époque, a conduit davantage vers une politique de minimisation plutôt que d'optimisation.

Sur la base d'une approche pas à pas, cas par cas, nous avons finalement réussi à retirer des zones contrôlées des réservoirs de stockage et des débris de démolition issus des bâtiments concernés. Depuis lors, la libération ou le rejet peut être considéré comme une pratique de routine, et bon nombre d'efforts ont été consacrés à l'optimisation liée au déclassement et aux méthodes et techniques de décontamination. Sur une base annuelle, plusieurs centaines de tonnes de matériaux décontaminés ont été éliminées par application du principe de libération.

D'un point de vue « coût-bénéfice », la libération apparaît indubitablement comme la méthode adéquate pour optimiser le volume des déchets radioactifs; à condition que les limites de rejet inconditionnel ne tendent pas vers la minimisation. La définition de limites de rejet inconditionnel devrait inclure les principes d'équité concernant les risques, les activités non nucléaires et les pratiques exemptées. La minimisation conduirait en effet à des investissement excessifs pour les activités de décontamination et les techniques de mesures. De plus, les mesures au niveau des limites de détection du matériel industriel disponible conduiraient à des résultats biaisés.

Cet article démontre 1'application du principe de libération dans trois cas concrets; il porte une attention particulière à la méthodologie de la décontamination, de l'échantillonnage, de l'analyse et de l'interprétation.

\section{Méthodologie de la libération et exemples concrets}

Initialement, à Belgoprocess la libération était fondée uniquement sur les mesures de contamination de surface. La méthode comportait deux mesures successives de la totalité $(100 \%)$ de la surface du matériel présenté. Les mesures étaient effectuées de manière indépendante par deux radioprotectionnistes qui utilisaient des instruments de mesures spécifiques. Tant que les matières sont présentes sur le site, un organisme de contrôle nucléaire indépendant dispose de la possibilité de procéder à des contrôles complémentaires. Pour des matériaux ayant été décontaminés, un délai de trois mois sépare les deux mesures. 
Le démantèlement progressant, le besoin se fit sentir d'étendre la procédure existant à d'autre types de mesures et, dès lors, d'adapter celle-ci à d'autres géométries de matériaux. Une étude portant sur les types de matières présentes dans les installations conduisit à quatre « types de libération », à savoir, les mesures de contamination de surface, les mesures de type «volumique » (par exemple le charbon actif en fûts), les mesures de matériaux stockés en fûts et les matières pouvant être homogénéisées. Pour chaque type, on a élaboré une méthodologie, établie chronologiquement, d'échantillonnages et de mesures indépendante des limites de rejet inconditionnel. Pratiquement tous les types de libération impliquent une combinaison de mesures de contamination surfacique et de mesures d'activité spécifique. En effet, des mesures d'activité spécifique (par exemple sur des échantillons centraux, sur des débris de béton, ...) peuvent donner des valeurs plus précises, complémentaires quant à l'activité résiduelle. Ces analyses peuvent également fournir une information relative à la pénétration possible de l'activité au sein des structures devant être mesurées.

À la suite de plusieurs années d'expériences, s'est manifestée la volonté de réduire la seconde mesure, pour la contamination surfacique, à $10 \%$ environ du matériau à mesurer. En effet, les quantités de matières déclarées comme contaminées audessus des niveaux de libération inconditionnelle lors de cette seconde mesure ne constituent qu'une fraction de pour cent de la quantité totale du matériau. De plus, dans la presque totalité des cas, les niveaux d'activité détectée étaient à peine supérieurs à ces limites.

De plus, un scénario similaire a été développé, pour considérer les aspects non nucléaires associés au retrait de matériaux du site. Dans ce scénario, référence est faite aux destinations potentielles du matériel impliqué.

Point important lors de la phase d'évaluation et particulièrement avant le déclassement, les données historiques portant sur les installations et les matériaux impliqués doivent être mentionnées. Toute l'information disponible devrait être collectée et étudiée en détail, puisqu'elle pourrait mettre en lumière l'évidence d'une contamination et/ou d'une décontamination antérieure d'installations ou structures ainsi que les changements dans l'équipement ou la modification des composants structurels et les types de techniques de décontamination utilisées (par exemple le nettoyage à eau sous pression ou le nettoyage à sec) tout au long de la vie de l'installation.

La méthodologie complète a été discutée avec l'organisme de contrôle indépendant ainsi qu'avec les autorités. À l'heure actuelle, les pratiques de libération inconditionnelle sont encore fondées sur une approche cas par cas, qui implique que les autorités (et/ou l'organisme de contrôle indépendant) doivent être consultées pour chaque nouveau type de matière susceptible d'être libérée. Lorsqu'on libère un type de matériau pour la première fois, la pratique et les niveaux de rejet retenus doivent être documentés et justifiés en fonction de la destination. À l'heure 
actuelle, les niveaux de libération inconditionnelle sont ceux mentionnés dans la publication officielle de l'AIEA (1996). Des écarts importants subsistent toutefois entre les études qui y sont citées.

\section{Cas $n^{\circ} 1$ : le classement des bâtiments $6 A$ et $6 B$ (Eurochemic)}

Deux petits bâtiments (6A/6B) de l'ancienne installation de retraitement Eurochemic ont été déclassés; ils constituent la première application à grande échelle du déclassement et de la libération en Belgique. Les bâtiments étaient utilisés pour le stockage de nitrate d'uranyle, de dioxyde de plutonium et des produits finaux des activités de retraitement. Les solvants utilisés y étaient également stockés. Le déclassement fut considéré comme un projet pilote visant à démontrer la faisabilité du déclassement d'installations nucléaires. Une attention particulière a été portée à la décontamination et à la libération (AIEA, 1995).

Les deux bâtiments, d'un volume total de $3300 \mathrm{~m}^{3}$, comportaient une surface de béton de $5000 \mathrm{~m}^{2}, 47$ tonnes de composants métalliques et $950 \mathrm{~m}^{3}$ de béton. La contamination surfacique moyenne était de l'ordre de quelques $\mathrm{Bq} / \mathrm{cm}^{2}$, avec des pics à $100 \mathrm{~Bq} / \mathrm{cm}^{2}$ en alpha et en bêta. Les débits de dose étaient compris entre 300 et $500 \mu \mathrm{Sv} / \mathrm{h}$.

Tous les équipements ont été retirés et les sols, murs et plafonds ont été décontaminés jusqu'au niveau du bruit de fond naturel de rayonnement. La décontamination des métaux a été réalisée par des procédés chimiques, par découpe et broyage, tandis que le béton fut décontaminé par bouchardage et par marteau pneumatique. Les réservoirs de stockage, présentant des contaminations relativement élevées en alpha et en bêta, ont été décontaminés jusqu'au niveau de rejets inconditionnels de $0,04 \mathrm{~Bq} / \mathrm{cm}^{2}$ pour les alphas et $0,4 \mathrm{~Bq} / \mathrm{cm}^{2}$ pour les bêtas. Le service de contrôle physique de 1'entreprise a autorisé le retrait des zones contrôlées de ces matériaux, avec l'accord d'un organe de contrôle indépendant.

La procédure de libération comportait :

- deux mesures successives de la totalité de la surface (100\%);

- des mesures de contrôle aléatoires effectuées par un organe de contrôle indépendant;

- la prise d'échantillons dans la masse et leurs analyses qualitative et quantitative;

- des analyses destructives sur des échantillons pris dans la masse montrant les plus hauts niveaux de radioactivité.

Deux agents du service de radioprotection ont effectué les mesures indépendamment l'un de l'autre. Ils ont employé des instruments de mesure retenus dans ce but. Aussi longtemps que les matériaux étaient présents sur le site, ils étaient à 
la disposition de l'organisme de contrôle indépendant, pour toute vérification supplémentaire.

La prise d'échantillons dans la masse était nécessaire pour faciliter l'approbation d'une libération inconditionnelle des débris de démolition des bâtiments. On a prélevé des échantillons des endroits où précédemment les pics d'activités les plus élevés avaient été détectés. On a estimé que, à ces endroits, la probabilité de pénétration de la contamination était la plus élevée. Toutes les analyses montrèrent que l'activité spécifique était bien inférieure au $\mathrm{Bq} / \mathrm{g}$, et était due uniquement à la présence de radionucléides naturels. Quoique la barrière psychologique quant à la libération ait été importante, pratiquement $65 \%$ de tous les métaux ont pu être libérés, la quantité restante ne présentant pas une géométrie autorisant les mesures. Au cours de nombreuses opérations, 76 tonnes de débris de béton ont été produites, dont $53 \%$ n'étaient pas radioactifs. Lors de la démolition finale des deux bâtiment, 2350 tonnes de tels débris furent portés dans une décharge publique pour matières inertes.

Le projet, démarré en 1988, conduisit au «green field » en 1991. Le problème majeur rencontré lors de l'application du principe de libération fut de convaincre les autorités d'accepter la pratique de la libération inconditionnelle. Par suite de l'absence de valeurs-guides pour la libération, les limites furent minimalisées mais certainement pas optimisées. Ainsi, les niveaux d'activité surfaciques ont été placés au niveau de la limite de détection de l'équipement de mesure existant. Ceci constitue la raison principale pour laquelle le département de contrôle physique contribua pour environ $16 \%$ aux coûts totaux engendrés par le déclassement des bâtiments $6 \mathrm{~A}$ et $6 \mathrm{~B}$.

\section{Cas $n^{\circ} 2$ : la fusion des dispositifs de stockage de combustible en acier inoxydable ( rack $»)$}

On a évalué divers scénarii pour le traitement, le conditionnement et la mise en décharge ou le recyclage de sept dispositifs de stockage de combustibles issus d'une centrale nucléaire REP (Teunckens et al., 1996; 1993). Les racks étaient en acier inoxydable et la masse totale atteignait 28 tonnes. Un rack se composait de 30 tubes d'une longueur de 3 mètres et d'une section de $(0,3 \times 0,3) \mathrm{m}^{2}$. Les données sur l'histoire de ces racks indiquaient qu'ils avaient été stockés dans une piscine à combustible mais n'avaient jamais été utilisés pour le stockage de combustible, suite à un défaut de construction. En accord avec les données comptables, on assigna une activité totale de $3,0 \mathrm{GBq}$ pour ces racks, correspondant à un niveau d'activité spécifique de $94 \mathrm{~Bq} / \mathrm{g}$. Une analyse coût-bénéfice indiqua que la fusion constituait le meilleur choix pour le traitement. C'est ainsi que, moyennant deux transports, les sept racks furent envoyés en Suède pour fusion dans la fonderie Studsvik. 
L'information de base à partir de laquelle les caractéristiques finales des racks ont été définies a été obtenue par une approche multiple, considérant des mesures par frottis (fraction non fixée), des échantillons et analyses de parties des racks, ainsi qu'une simulation numérique fondée sur les mesures de débits de dose et des données relatives à la contamination surfacique.

L'analyse des échantillons issus des racks présentant les débits de dose les plus élevés révéla que ${ }^{60} \mathrm{Co}$ était le constituant majeur (77\%), suivi par ${ }^{137} \mathrm{Cs}(20,5 \%)$, ${ }^{241} \mathrm{Am}(1,4 \%)$ et ${ }^{94} \mathrm{Nb}(1,1 \%)$. L'abrasion et la décontamination électrochimique de ces échantillons démontrèrent que seule une partie de la contamination pouvait être éliminée. En conséquence, et parce que du ${ }^{94} \mathrm{Nb}$ fut détecté, il a été considéré que l'activité pouvait être due à une activation des structures. Différentes estimations indiquèrent une activité spécifique de l'ordre de 1,8 à $7 \mathrm{~Bq} / \mathrm{g}$. Par simulation numérique sur ordinateur, on obtint une activité spécifique de $4 \mathrm{~Bq} / \mathrm{g}$ pour le rack présentant le débit de dose le plus élevé et cette valeur fut considérée comme valeur la plus probable.

Suivant les données obtenues après la première campagne de fusion ( 16 tonnes), on trouva que l'activité initiale de ces racks était d'environ $10 \mathrm{~Bq} / \mathrm{g}$ en ${ }^{60} \mathrm{Co}$. Cette valeur fut déduite de l'analyse d'échantillons prélevés sur les lingots, les scories et les poussières. Il apparut que l'activité spécifique en alpha et en bêta avait été sous-estimée d'un facteur de 6,7 et 4,4 respectivement.

Bien que toutes les mesures de caractérisation aient été réalisées de manière parfaite, des difficultés majeures ont été rencontrées, telles que :

- le manque de données historiques sur la caractérisation et l'utilisation des racks (contamination ou activation);

- la représentativité de l'échantillonnage (tests par frottis, échantillons découpés), due principalement aux géométries complexes;

- les erreurs possibles lors de l'interprétation des données de contamination surfacique, considérant la quantité inconnue de la contamination transférable et l'incertitude sur sa distribution quant à l'homogénéisation.

Apparemment, il n'existe pas non plus de relation linéaire entre les débits de dose mesurés initialement et les activités spécifiques obtenues après analyse des échantillons de lingots. On peut aussi se demander, et principalement pour les raisons mentionnées plus haut, si la fusion de grandes quantités d'échantillons des racks à l'échelle du laboratoire aurait conduit à une meilleure estimation. D'autre part, la fusion a prouvé qu'elle est la meilleure option en ce qui concerne la réduction des volumes et l'obtention de données d'activités fiables, pour autant toutefois que l'échantillonnage et les analyses soient réalisés en utilisant des techniques/procédures qualifiées et certifiées par les partis impliqués. 
En définitive, puisque la quasi-totalité (>95\%) de la radioactivité résiduelle était due au ${ }^{60} \mathrm{Co}$, le matériel sera libéré pour réutilisation, après une période intermédiaire de stockage permettant sa décroissance radioactive.

\section{Cas $n^{\circ} 3$ : fusion de l'aluminium provenant d'échangeurs de chaleur}

Comme pour les dispositifs de stockage de combustible, on a évalué plusieurs scénarii pour le traitement, le conditionnement et la mise en décharge ou le recyclage de neuf échangeurs de chaleur d'aluminium (type horizontal), d'une masse de 42 tonnes. De plus, on y a ajouté deux tonnes de tubes d'aluminium. La contamination fut considérée comme présente sur la surface interne des tubes et non sur la surface de l'enveloppe des échangeurs. Se fondant sur l'histoire, on n'a attribué aucune activité spécifique aux échangeurs de chaleur.

À partir d'évaluations techniques et économiques, on considéra que la fusion est la meilleure option pour le traitement. Avant fusion, toutefois, un nettoyage sous vide des échangeurs a été effectué en vue d'éliminer les fragments libres. Par des frottis, prélevés après ce nettoyage, on estima l'activité, moyennée sur la masse totale des échangeurs, à environ $3 \mathrm{~Bq} / \mathrm{g}$ pour les alphas et à $12 \mathrm{~Bq} / \mathrm{g}$ pour les bêtas. Pour les tubes, on obtint des valeurs plus élevées de $49 \mathrm{~Bq} / \mathrm{g}$ en alpha et $98 \mathrm{~Bq} / \mathrm{g}$ en bêta. Ces valeurs pourraient toutefois être réduites d'un facteur 2 à 10 par décontamination au jet d'eau à haute pression.

Les données recueillies après la fusion des racks, (voir le paragraphe 4), ont toutefois conduit à réévaluer les estimations mentionnées plus haut. Ainsi, 6 échantillons furent prélevés des tubes provenant des parties supérieures et inférieures de trois échangeurs. Les mesures directes de ces échantillons indiquèrent des niveaux d'activité de $29,9 \mathrm{~Bq} / \mathrm{g}$ et $26 \mathrm{~Bq} / \mathrm{g}$. Ces valeurs apparaissent comme nettement plus élevées que celles dérivées des mesures de frottis (facteur 10 en alpha, 2 en bêta).

Après la fusion d'échantillons par Studsvik en Suède, les lingots et scories furent analysés, on obtint des niveaux d'activité de $74,2 \mathrm{~Bq} / \mathrm{g}$ en alpha et $7,1 \mathrm{~Bq} / \mathrm{g}$ pour le ${ }^{60} \mathrm{Co}$. Sur cette base, une réévaluation de l'activité moyennée sur la masse totale des échangeurs conduisit à $0,2 \mathrm{~Bq} / \mathrm{g}$ en bêtas $\left({ }^{60} \mathrm{Co}\right)$ et $1,9 \mathrm{~Bq} / \mathrm{g}$ en alphas.

Cette dernière valeur est supérieure à la limite de rejet inconditionnel en Suède, (1 Bq/g) et les échangeurs furent nettoyés mécaniquement à la brosse d'acier et sous vide puis rincés à l'eau pour éliminer les particules et poussières de la surface. Après nettoyage, un premier transport de 4 échangeurs (18,5 tonnes) fut effectué vers la Suède. Lors de la préparation de ce transport, plusieurs difficultés se présentèrent quant à la définition exacte des matières à envoyer. Des contacts permanents entre les autorités des pays concernés conduisirent finalement à classer ces matières comme marchandises ré-utilisables. La résolution de ce différent demanda pratiquement 6 mois. 
Dans l'usine de fusion contrôlée de Studsvik, les échangeurs furent fondus en 33 lots séparés. Les matériaux de l'enveloppe et les tubes furent traités séparément et des échantillons furent prélevés pour analyse. Des échantillons des lingots provenant de la fusion des parties extérieures des échangeurs indiquèrent des niveaux d'activité spécifique en alpha et en bêta bien inférieurs aux limites de rejet inconditionnel. Dès lors, ce matériel était acceptable pour le rejet inconditionnel en Suède. La fusion des tubes internes donna cependant des lingots dont l'activité en ${ }^{241} \mathrm{Am}$ était légèrement supérieure aux limites, du moins pour certains lots. Il en fut ainsi également pour ${ }^{60} \mathrm{Co}$, quoique ceci ne doive pas être considéré comme un problème puisque le stockage de décroissance est considéré comme une phase acceptable pour cette pratique. Si le matériel avait été fondu, sans séparation des tubes internes et de l'enveloppe externe des échangeurs, les concentrations en activité auraient été de $0,42 \mathrm{~Bq} / \mathrm{g}$ pour ${ }^{241} \mathrm{Am}$ et de $0,78 \mathrm{~Bq} / \mathrm{g}$ pour ${ }^{60} \mathrm{Co}$. La gammaspectrométrie indiqua que ${ }^{60} \mathrm{Co}$ était le radionucléide le plus abondant (>98\%).

L'analyse de quelques échantillons de lingots issus des tubes montra toutefois un rapport ${ }^{238} \mathrm{Pu}{ }^{241} \mathrm{Am}$ égal à 4 . Par conséquent, il était évident que l'activité totale en alpha dépassait largement les limites de rejet inconditionnel. C'est pourquoi, ces lingots ne furent pas considérés comme libérables et furent renvoyés à BP en Belgique.

Suite à ces expériences, on étudia une décontamination mécanique « agressive » des échangeurs. Ainsi, les échangeurs restants furent décontaminés mécaniquement à l'aide d'un tuyau nettoyeur à air disposant d'une tête de nettoyage fixée sur une pièce rotative équipée d'un jet d'eau pour éliminer les dépôts lorsqu'ils se produisaient.

Avant la planification de transports supplémentaires d'échangeurs pour fusion, et par suite des écarts entre les données analytiques et les valeurs estimées, on mit en œuvre un programme de recherche à l'échelle du laboratoire, dans le but de lever certaines incertitudes associées aux échantillonnages et aux analyses. De plus, après décontamination, des échantillons furent prélevés des tubes des échangeurs aux fins d'analyse, à la fois par des laboratoires de BP et de Studsvik. L'objectif était de caractériser complètement ces échantillons avant et après la fusion. Plus précisément, le but était d'obtenir une procédure d'échantillonnage et d'analyse pouvant être approuvée et acceptée par toutes les parties impliquées; c'était une priorité puisque des «mésinterprétations» peuvent conduire à une fusion et/ou à des opérations de décontamination inutiles. En effet, au vu des coûts potentiels associés, d'autres méthodes de traitement pouvaient devenir plus intéressantes (par exemple, la découpe, le compactage et le cimentage comme pour les déchets nucléaires). Les données des deux laboratoires ne sont, à ce jour, pas encore disponibles ou n'ont pas encore pu être échangées et comparées. On ne peut donc pas tirer de conclusions.

Comme indiqué, aucun renvoi d'échangeurs restants ne pourra être envisagé aussi longtemps qu'un accord n'aura pas été obtenu quant à la méthodologie d'échantillonnage et d'analyse, et à l'interprétation des données obtenues. 
Ce cas illustre toutefois clairement les difficultés rencontrées pour ce qui concerne la représentativité de l'échantillonnage, les mesures et leur interprétation. Ceci est illustré dans le tableau I reprenant les diverses valeurs des concentrations d'activités telles que calculées et mesurées actuellement.

\section{TABLEAU I}

Valeurs des concentrations de l'activité calculées et mesurées (Bq/g). Survey of calculated and measured activity concentrations $(B q / g)$.

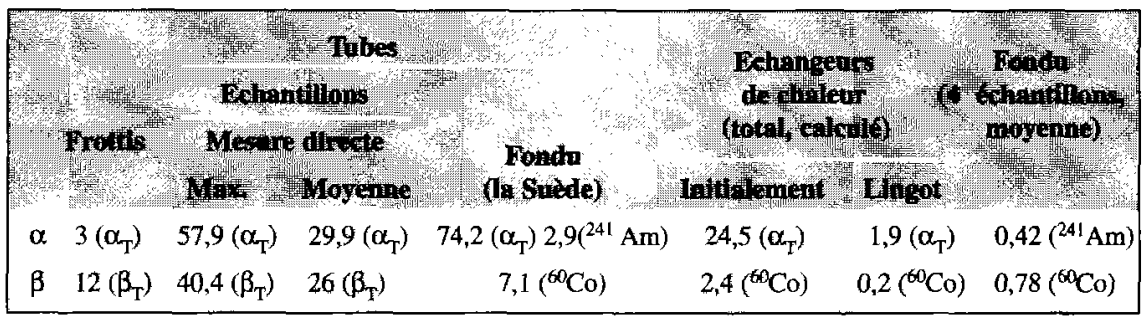

\section{Observations pratiques concernant les mesures de libération}

Le tableau II présente un survol des données caractéristiques pour les cas mentionnés ici. Le tableau III fournit des données sur la libération inconditionnelle de matériaux résultants du déclassement de bâtiments de l'ancienne usine de retraitement Eurochemic. Le tableau III démontre clairement l'importance de l'application des pratiques de libération pour l'optimisation des volumes de déchets radioactifs. Quelques constatations pratiques résultent de l'expérience acquise en matière de mesure de libération :

- il est important de disposer de données historiques concernant la composition en radionucléides et l'utilisation des matériaux concernés; des données relatives à des manipulations antérieures comme la décontamination sont aussi extrêmement importantes ;

- les matériaux et composants de structure doivent être débarrassés de toutes poussières afin d'éviter une recontamination ;

- après décontamination chimique, un certain délai sera prévu afin de permettre le « ressuage » du matériau;

- les mesures doivent être effectuées dans des endroits où le bruit de fond naturel est faible;

- les mesures directes pour la contamination alpha sont très sensibles à l'état des surfaces (par exemple, des surfaces fortement bouchardées) et à la présence de poussières ou de sels; la surface doit être complètement sèche et, sur ce point, il est extrêmement important d'utiliser des techniques de 
TABLEAU II

Résumé des cas.

Summary of cases.

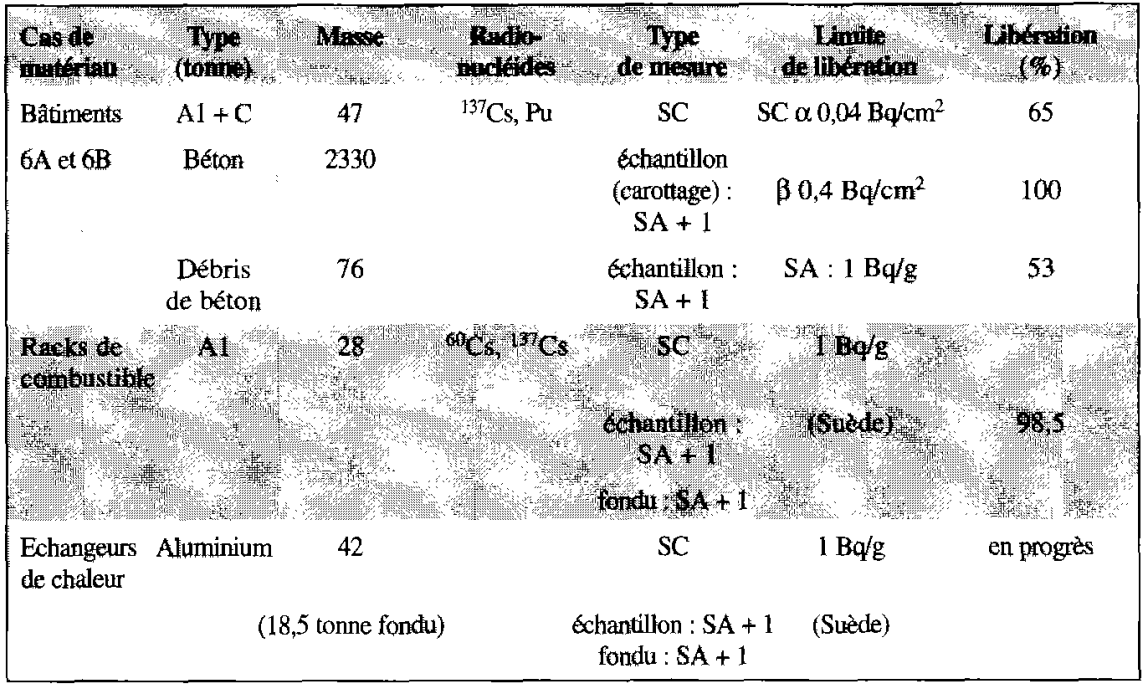

SC: Contamination de surface $\quad$ AI: Acier inoxydable $\quad$ SA: Activité spécifque $\quad C:$ Acier au carbone I: Composition isotopique

TABLEAU III

Démantèlement du bâtiment de retraitement (Eurochemic). Quantités de produits générés. Decommissioning Eurochemic of reprocessing building. Mass of generated products.

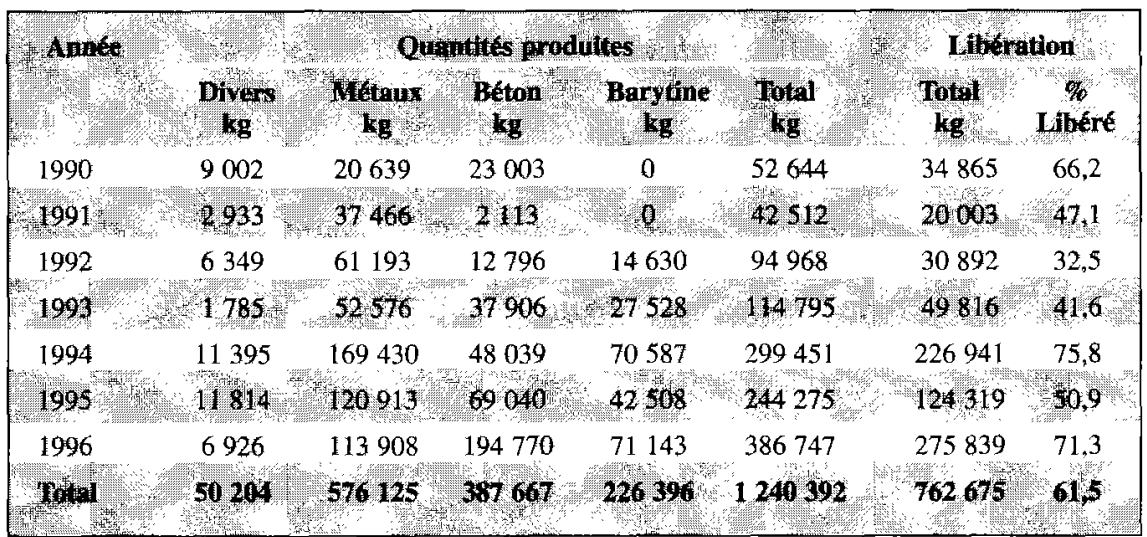


décontamination à sec conduisant à des surfaces lisses (par exemple pour le décapage de murs de béton);

- la mesure des fûts par balayage est très souhaitable pour les radionucléides émetteurs gamma, pour autant que le matériau soit distribué de manière homogène; des erreurs parfois importantes peuvent être induites pour d'autres types de matériaux : ceci provient de la correction inadéquate apportée pour les effets de blindage;

- dans les installations de retraitement, les émetteurs alpha sont généralement associés à des émetteurs bêta et gamma ; la composition initiale en radionucléides peut changer très fortement, par exemple en cas de rinçages nombreux du matériau. En ce sens, le soin le plus grand sera apporté dans le calcul des activités alpha à partir des activités détectées;

- des étalons certifiés devront être utilisés dans les géométries souhaitées et représentatives;

- par suite des faibles valeurs de limites de rejet inconditionnel proposées, les incertitudes sur les résultats analytiques peuvent devenir très importantes;

- les techniques d'analyse neutronique active-passive conduisent à des limites de détection pour les émetteurs alpha beaucoup trop élevées dans le cadre de la libération;

- un balayage de l'émission gamma in situ semble très prometteur pour les mesures de surfaces importantes; combinée avec l'échantillonnage et l'analyse des débris de démolition représentatifs, cette technique peut fournir des résultats valables et fiables et, en tant que telle, peut être considérée comme l'une des techniques d'aide à la prise de décision avant démolition;

- après libération et avant mise en dépôt, les matériaux doivent être entreposés dans des endroits spécifiquement réservés;

- en cas de rejet inconditionnel de matériaux, les aspects non-nucléaires sont tout aussi importants, particulièrement lors de la mise en dépôt dans des décharges publiques; ces dernières sont soumises à une législation spécifique comportant des exigences particulières édictées par des autorités compétentes différentes ;

- la réglementation variant d'un pays à l'autre, il se pourrait que du matériel libéré dans un pays soit considéré comme radioactif lorsqu'il est exporté vers un autre.

\section{Conclusions}

Belgoprocess applique le concept de libération en vue d'optimiser le volume des déchets radioactifs. Il rejette annuellement plusieurs centaines de tonnes de matériaux hors des zones contrôlées. Cette quantité est en progression constante par suite de l'utilisation, lors du démantèlement et de la décontamination, de méthodes et d'outils optimalisés. 
Bon nombre de problèmes rencontrés dans les pratiques de libération sont d'avantage de nature psychologique que d'ordre technique. Les pratiques industrielles qui existent depuis longtemps connaissent moins ce phénomène, car le public accepte ces pratiques en tant que telles. Le public n'a pas connaissance de la valeur actuelle du facteur de risque.

Nombre d'études sur l'exemption/libération sont fondées sur des résultats obtenus dans des secteurs d'activités différents, comportant potentiellement l'introduction d'erreurs multiples lors de la proposition de limites de rejet inconditionnel. Ceci va conduire à des valeurs extrêmement conservatives. Ceci peut être illustré par la dose individuelle de 10 microsieverts par an pour une pratique de rejet; elle est considérée à ce jour comme le maximum acceptable. Les limites de rejet inconditionnel fondées sur cette philosophie n' ont plus rien à voir avec l'optimisation. Des valeurs trop restrictives imposent des limitations sur les pratiques de la libération. Les limites doivent être fondées sur des facteurs de risque prenant en compte le principe d'équité eu égard aux autres industries et/ou pratiques existantes, acceptées par le public, et en gardant à l'esprit l'usage optimal des ressources. Les facteurs de risque pour ces pratiques sont largement supérieurs à ceux couramment retenus dans la plupart des évaluations de sûreté relatives à la libération de matière.

Les études de libération devraient, de plus, être attentivement examinées, en ce qui concerne les points de départ et la définition correcte des paramètres. L'implication de praticiens lors de ces études, avant la publication des conclusions finales, devrait certainement être très bénéfique, comme riche d'apports et d'encouragements.

\section{Références}

AIEA (1996) Clearance levels for radionuclides in solid materials. Aplication of exception principes, TECDOC-855, Vienne, Autriche.

Teunckens L., Blommaert W. (1993) Radiological Characterisation of Metal Components in View of Melting, Colloque « Experiences in the Application of Exemption Principles », Agence internationale de l'énergie atomique, Vienne, Autriche, 2-4 Novembre 1993, IAEA-TECDOC-807.

Teunckens L., Lewandowski P., Dresselears R., Walthery R., Andersson O., Menon S., Bjerler J. (1994) Economical Aspects of Melting and/or Recycling of Waste Metals from Decommissioning, Seminar on Melting and Recycling of Metallic Waste from Decommissioning, Krefeld, Allemagne, 27-29 Octobre 1993, European Commission, Nuclear Science and Technology, EUR $15691 \mathrm{EN}$.

Teunckens L., Reynders R., Blommaert W., Baekelandt L. (1993) Decommissioning of Final Product Storage Buildings at the Former Eurochemic Reprocessing Plant, Colloque « Experiences in the Application of Exemption Principles », Agence Internationale de l'énergie atomique, Vienne, Autriche, 2-4 Novembre 1993, AIEA-TECDOC-807, juillet 1995. 